\title{
Macroscopic Findings Reference Identifier
}

National Cancer Institute

\section{Source}

National Cancer Institute. Macroscopic Findings Reference Identifier. NCI Thesaurus.

Code C119866.

A sequence of characters used to identify, name, or characterize the macroscopic findings reference. 